\title{
Alternative kulturelle Einrichtungen in deutschen Städten und Gemeinden. Ein Beitrag zur Kritik ökonomischer Perspektiven auf Kreativräume
}

\author{
Christoph Mager
}

Eingegangen: 9. Januar 2014 / Angenommen: 8. September 2014 / Online publiziert: 17. Oktober 2014

(C) Springer-Verlag Berlin Heidelberg 2014

\begin{abstract}
Zusammenfassung Mit der umfassenden Bestandsaufnahme im Enquetebericht des Deutschen Bundestages „Kultur in Deutschland" von 2007 rücken neben öffentlichen und privatwirtschaftlichen Infrastrukturen auch zunehmend alternative Einrichtungen des zivilgesellschaftlichen Kultursektors in das Blickfeld von Kulturpolitik, Raumplanung und Kreativwirtschaft. Der Beitrag argumentiert am Beispiel der materiellen und diskursiven Entwicklungen soziokultureller Zentren seit den 1960er Jahren, dass kultur- und kreativwirtschaftliche Perspektiven zentrale Dimensionen kultureller Infrastrukturen systematisch ausblenden. Erstens implizieren berufsbezogene Abgrenzungen von Kreativität eine qualitative Trennung von professionellen, wertschöpfenden Tätigkeiten einerseits und laienhaften, profanen Praktiken andererseits. Eine Folge ist die Hierarchisierung der jeweiligen Sozialräume. Zweitens wird kulturelle Kreativität aktualitätsbezogen auf ökonomisch verwertbare Praktiken reduziert, was die historischen Entwicklungslinien kulturpolitischer Diskussionen um Frei- und Spielräume für eine demokratische und emanzipierte Teilhabe an Kultur verdeckt. Drittens unterschätzt ein bevorzugt innerstädtischer Fokus die Rolle von Kultureinrichtungen im suburbanen Raum und auf dem Lande, deren zivilgesellschaftliche Formen dort wichtige und die oftmals einzigen Ankerpunkte für kreative Praktiken darstellen. Die Diskussion zeigt, dass ein rein ökonomischer Fokus auf
\end{abstract}

Dr. C. Mager $(\bowtie)$

Institut für Geographie und Geoökologie, Karlsruher Institut für Technologie,

Reinhard-Baumeister-Platz 1,

76131 Karlsruhe, Deutschland

E-Mail: christoph.mager@kit.edu kulturelle Infrastrukturen dazu tendiert, historisch, funktional und sozialräumlich selektiv zu argumentieren.

Schlüsselwörter Kulturelle Infrastruktur · Kreativität · Kreativwirtschaft · Kulturpolitik · Soziokultur · Deutschland

\section{Alternative Cultural Institutions in Germany: Questioning Economic Perspectives on Spaces of Creativity}

\begin{abstract}
In 2007 the German Bundestag issued an extensive parliamentary report on "Culture in Germany" that spurred broad debates about the role of cultural institutions in urban and regional planning. Subsequently, these infrastructures gained particular importance in discussions on the cultural and creative industries as nodes for professional activities and marketplaces for cultural goods. By analyzing the material and discursive trajectories of alternative cultural and community centres in Germany the paper argues that economic perspectives on spaces of creativity obscure important dimensions of creative practices. Firstly, conceptions of creativity as a defining feature of occupations and economic sectors imply that creative pursuits are ultimately determined by patterns of supply and demand. Mundane activities on a day-to-day basis however show that alternative creativities are frequently driven by voluntary engagements, personal desires and affection towards people and ideas. Secondly, as studies on the creative industries offer rather current diagnoses of cultural infrastructures the historical dimension of political discussions is often missing from theses analyses. Established since the 1960s, alternative cultural institutions are conceptualized as sustainable meeting places that offer spaces for
\end{abstract}


communication, self-determination and free play. Finally, creative industry thinking emphasizes specific geographical contexts for creative production. The overriding observation is one of urban agglomeration and spatial clustering. The mapping of alternative cultural infrastructures reveals a more complex and nuanced geography of non-profit creativity that encompasses material spaces in suburban and more rural areas. Cultural and community centres offer an important and sometimes occasional anchor point for creativity in these peripheries lacking publicly funded and privately financed cultural infrastructures.

Keywords Cultural infrastructure $\cdot$ Creativity $\cdot$ Cultural policy $\cdot$ Alternative culture $\cdot$ Cultural centre $\cdot$ Germany

\section{Einleitung}

Nach den Strukturberichten des Verbandes deutscher Städtestatistiker zu Kultur und Bildung aus den Jahren 1987 und 1998 rücken kulturelle Infrastrukturen mit der umfassenden Bestandsaufnahme im Enquetebericht des Deutschen Bundestages „Kultur in Deutschland“ (Enquete-Kommission 2007) wieder verstärkt in das Blickfeld von Kulturpolitik und räumlicher Planung (von Beyme 2012: 186 ff.). Bislang wurde die kulturelle Ausstattung von Städten und Gemeinden in raumbezogenen Studien entweder als ein Indikator für Zentralität (Christaller 1968: 139), als ein Standort-, Image- und Beschäftigungsfaktor urbaner Ökonomie (Taubmann 1987; Behr/Gnad/Kunzmann 1989) oder als ein Bestandteil von Urbanität hervorgehoben (Jacobs 1961). Wirtschaftsgeographische Studien untersuchen die Rolle kultureller Einrichtungen zudem als Teil spezifischer sozioökonomischer Organisationsformen. In kreativen Clustern oder urbanen Kulturdistrikten konzentrieren sich kulturbezogene und kreative Akteure, deren Tätigkeiten sich durch Innovations- und Experimentierfreudigkeit auszeichnen. Hier entstehen spezifische Ökologien, in denen unter Bedingungen von Befristung, Unsicherheit und Risiko mithilfe kontextbezogener Regeln und Institutionen ein vertrauensvoller Austausch von ökonomisch relevantem Wissen vollzogen werden kann. Diese engen Beziehungen verlaufen häufig entlang von Wertschöpfungsketten und -netzwerken, für die kulturelle Einrichtungen entscheidende Produktions-, Konsum- und Marktorte darstellen können (Watson/ Hoyler/Mager 2009; Lange/Bürkner 2010).

Andere Diskussionen sprechen kulturellen Einrichtungen wie Museen, Theatern und Clubs sowie den Ausbildungsund Qualifizierungseinrichtungen kultureller und kreativer Berufsfelder als Teil eines städtischen ,creative field“ (Scott 2010) eine wichtige Bedeutung für die Ansiedlung und die Standortpersistenz der Kultur- und Kreativwirtschaft zu (MWMTV 1998). Eine viel diskutierte Perspektive betont beispielsweise die durch Kultureinrichtungen geschaffenen Möglichkeiten, vielfältige städtische Unterhaltungsangebote zu konsumieren und individuelle Lebensstile zu pflegen. Städte und Metropolregionen werden so zu toleranten, abwechslungsreichen und attraktiven Orten, deren spezifische kulturelle Szenen und Konsumangebote eine zentrale Voraussetzung für die Zuwanderung hochqualifizierter und kreativer Arbeitskräfte darstellen (Florida 2002). Da das Vorhandensein und der Zuwachs von Arbeitskräften der Kulturökonomie und Kreativwirtschaft dabei gewissermaßen als einzig probates Mittel einer erfolgreichen und zeitgemäßen Stadtentwicklungs- und Regionalwirtschaftspolitik gelten, fällt den kulturellen Infrastrukturen die Aufgabe zu, diese ökonomischen Praktiken zu bündeln und Räume für Kreativität zur Verfügung zu stellen.

Die Systematisierung und Abgrenzung von Kultur und Kreativität stellt eine zentrale wissenschaftliche Aufgabe dar, um räumlich und zeitlich vergleichende Grundlagen für Planungsüberlegungen zu schaffen. Anhand von Parametern der amtlichen Statistik werden spezifischen Sektoren, Wirtschaftszweigen, Teilbereichen oder Berufen die Attribute kreativ oder innovativ zugesprochen (vgl. Mossig 2009). Als Ergebnis einer raumstatistischen Analyse lassen sich kultur- und kreativwirtschaftliche Zentren und Peripherien auf verschiedenen administrativen Ebenen identifizieren und vergleichen. Für den deutschsprachigen Kontext liegt mittlerweile eine Vielzahl sogenannter Kultur- oder Kreativwirtschaftsberichte vor, die als Auftragsstudien Bestandsaufnahmen solchermaßen ökonomisch vermittelter Kreativität auf der Ebene von einzelnen Städten, Ländern und dem Bund vornehmen (für eine Übersicht vgl. Glückler/ Ries/Schmid 2010). Das Gros dieser Untersuchungen arbeitet mit vergleichenden Daten, die der Definition von Kulturund Kreativwirtschaft der Wirtschaftsministerkonferenz der Länder und der Enquete-Kommission des Deutschen Bundestages folgen (vgl. Enquete-Kommission 2007) und für den Kulturbereich zunächst eine einfache Unterscheidung in einen öffentlich geförderten und einen privatwirtschaftlich organisierten Sektor zugrunde legen. Institutionell zählen zum öffentlichen oder staatlichen Sektor kulturelle Einrichtungen wie Theater, Museen, Opernhäuser oder Bibliotheken, der private Sektor umfasst unter anderem die kulturwirtschaftliche Aktivität des Musikmarkts, der Filmwirtschaft, des Literatur- und Kunstmarkts sowie die Werbe-, Software- und Medien-Informationsbranche. Einzelne Systematisierungen folgen dem für die schweizerische Kulturwirtschaft entwickelten sogenannten Drei-SektorenModell (Weckerle/Söndermann/HfGK 2003), das auf der Basis von Trägerschaft und Finanzierungsart zusätzlich einen sogenannten intermediären Kultursektor ausweist, der zivilgesellschaftlich organisiert ist. $\mathrm{Zu}$ diesem zählen Mäzene, gemeinnützige Organisationen wie Kulturstiftungen, Kunst- und Kulturvereinigungen, Amateurensembles 
von Musik- und Theatervereinen sowie Einrichtungen der Alternativ- und Soziokultur. Im Verständnis des trisektoralen Modells finden künstlerische und kulturelle Produktion in sich überschneidenden öffentlichen, privatwirtschaftlichen und privatrechtlich-gemeinnützigen Bereichen statt.

Obschon diese Organisationsformen der Kultur- und Kreativwirtschaft ,weltweit in dieser Ausprägung in Deutschland einmalig" sind (Der Beauftragte der Bundesregierung für Kultur und Medien 2012: 8), erfolgt die Anerkennung des intermediären Sektors in Studien zur Kultur- und Kreativwirtschaft bislang nur ausnahmsweise. Der vorliegende Beitrag unternimmt den Versuch, die Vielfalt alltäglicher Kreativität in verschiedenen kulturpolitischen und geographischen Kontexten zu dokumentieren und zu analysieren. Es soll im Folgenden darum gehen, für deutsche Städte und Gemeinden die Bedeutung von jenen kulturellen Infrastrukturen als Kreativräume abzuschätzen, die sich seit den kulturpolitischen Diskussionen der 1960er Jahre um eine Demokratisierung kultureller Teilhabe als Alternativen zu den institutionalisierten Orten traditioneller hochkultureller Kulturvermittlung herausgebildet haben (Glaser/Stahl 1983). Diese Einrichtungen sehen sich heute konfrontiert mit vielfältigen Erwartungen, sowohl gemeinnützige Kulturarbeit zu leisten, als auch Arbeits- und Aufführungsorte für Kreative zu bieten. Im Mittelpunkt stehen soziokulturelle Einrichtungen, die als geographische Orte einen materiellen, sozialen und diskursiven Rahmen für kulturelle und kreative Praktiken bilden. Ausgehend von Implikationen der kulturalistischen Wende in den Sozial- und Wirtschaftswissenschaften (vgl. Gibson 2012a) sowie von Forderungen, Kultur und Kreativität in ihren jeweiligen Kontexten zu verorten (Pratt 2011), folgt die Argumentation neueren Arbeiten, die Kultur und Kreativität auch jenseits dominanter ökonomischer Logiken städtischer Kreativwirtschaft verstehen wollen (Edensor/Leslie/Millington et al. 2010). Die historische Dimension kulturpolitischer Diskussionen, die zur Etablierung alternativer Einrichtungen wie Bürgerhäusern, Jugendkunstschulen und soziokulturellen Zentren geführt haben, eröffnet einen neuen Blick auf die Geographie von Kreativität und trägt zu einer Neu-Kontextualisierung von Kreativräumen bei.

Zunächst referiert dieser Beitrag aktuelle Ansätze und kritische Perspektiven auf kulturelle Einrichtungen, die als materielle und soziale Infrastrukturen Bezugs- und Ankerpunkte kultureller und kreativer Betätigung darstellen. Kreativität wird dabei nicht ausschließlich als Eigenschaft von Berufen und Produkten gefasst, sondern als alltäglicher, disperser, pfadabhängiger und nicht immer zweckgerichteter Prozess des Improvisierens, Schöpfens und Hervorbringens, der den dominant ökonomischen Charakter überwindet. Ein zweiter Schritt ordnet alternative kulturelle Einrichtungen und ihre Trägergruppen in Diskussionen der vergangenen Jahrzehnte ein und fragt, welche Bedeutung ihnen kulturpo- litisch, sozialpolitisch und in der alltäglichen Kulturarbeit beigemessen wurde. Es zeigt sich, dass diese Kreativräume weniger der wirtschaftlichen Herstellung von Produkten dienen, sondern als kontextbezogene Ressourcen Möglichkeiten zur kreativen Gestaltung des Selbst bieten können. Die Ergebnisse einer empirischen Erhebung zu soziokulturellen Zentren und Initiativen in Deutschland decken abschließend die bislang weitgehend unsichtbare und vielfältige Geographie solcher alternativen Kreativräume auf.

\section{Kreativräume jenseits der Kreativwirtschaftsdebatten}

Debatten um die Kreativwirtschaft in Deutschland haben ihren Ausgangspunkt in Arbeiten zur städtischen Kulturwirtschaft, die seit den 1980er Jahren in Kontexten der Raumplanung und Stadtgeographie entstanden sind (Kunzmann 1992). Den Hintergrund boten unter anderem die grundlegenden sozioökonomischen Prozesse der De-Industrialisierung sowie die Neuausrichtung von Stadtpolitik entlang spätmoderner Entscheidungs- und Steuerungsformen (vgl. Häußermann/Läpple/Siebel 2008). Kultur galt als Hoffnungsträgerin, die den sektoralen Wandel hin zu einer Wissens- und Dienstleistungsgesellschaft mittragen und zugleich Angebote für eine zunehmend erlebnis- und konsumorientierte Gesellschaft unterbreiten sollte. In Nordamerika macht seit Beginn des vergangenen Jahrzehnts die These der sogenannten kreativen Klasse weit verbreitet Hoffnung, durch die Schaffung geeigneter Rahmenbedingungen Prozesse der Stadt- und Wirtschaftsentwicklung positiv beeinflussen und die Attraktivität städtischer Standorte erhöhen zu können (Florida 2002). Vertreter hochqualifizierter und künstlerischer Berufsgruppen orientieren sich bei ihren Wanderungsentscheidungen demnach weniger am vorhandenen Arbeitsplatzangebot als vielmehr an kulturell differenzierten und toleranten Umwelten ,kreativer Städte", in denen sie sich vorstellen können zu leben und zu arbeiten. Folgt man dieser Logik, ist die Bereitstellung kultureller Angebote und kultureller Infrastruktur Teil eines normativen Skripts, das den Zuzug kreativer Personen und ökonomisches Wachstum zum Ziel hat (Peck 2005; Krätke 2011).

Im wissenschaftshistorischen Zusammenhang lässt sich das gesteigerte Interesse an der Kultur- und Kreativwirtschaft mit einer Entwicklung seit den 1990er Jahren in Verbindung bringen, die Kultur und Ökonomie nicht länger als getrennte und quasi-natürliche Ordnungskategorien versteht, sondern auf die Komplementarität und Einbettung dieser diskursiv erzeugten Elemente in einem hybriden Ganzen hinweist (Castree 2004). Eine erneuerte Wirtschaftsgeographie öffnet sich im Rahmen einer kulturalistischen Wende stärker sozial- und kulturgeographischen Ansätzen und reflektiert sowohl Prozesse der ökonomischen 
Vermittlung von Kultur als auch die kulturelle Einbettung ökonomischen Handelns (Crang 1997). Insgesamt propagiert eine ,andere Geographie“ des Ökonomischen (Berndt/ Glückler 2006) die Erweiterung struktureller und institutioneller Ansätze um akteurs-, praxis- und körperbezogene Betrachtungsweisen (Amin/Thrift 2007), ohne Fragen nach sozialen Prozessen und materiellen Orten auszuklammern. Die aktuelle „second wave of research on the cultural and creative industries" (Gibson 2012a: 284) will die Debatte unter anderem um eine differenzierte materielle Verortung von kreativen Akteuren und sozialen Praktiken und um die prozessuale Perspektive auf die Vielfalt alltäglicher Formen von Kreativität bereichern.

\subsection{Substrat und Konstruktion: materielle Räume kreativer Praktiken}

Orte gelten als eine fundamentale Einflussgröße auf Kreativität (Gibson 2003; Pratt 2008). Materielle, technologische, organisatorische und affektive Merkmale eines Ortes schlagen sich in kreativen Praktiken und Artefakten nieder, die sich in anderen Kontexten nie vollständig replizieren lassen (Scott 2010: 124). Materielle Kreativräume unterliegen zunächst klassischen Logiken von Ökonomie. Sie müssen für Akteure zugänglich und erschwinglich sein und in einer gewissen Nähe zu ausreichenden Absatzmärkten liegen. Gebaute Umwelt dient als physische Grundlage für Kreativität - Quartiere, Gebäude, Räume, Bühnen, Tische, Sitzgelegenheiten. Architekturen, die Nutzungsstruktur von Gebäuden und die Zugänglichkeit von Räumen beeinflussen als Teile der urbanen Landschaft Potenziale und Ergebnisse künstlerischer Praktiken. Kulturelle Einrichtungen prägen als gebaute Umwelt die Qualitäten des öffentlichen Raums und die Charakteristika von Orten, die als visuelle Reize und Teil einer historischen Umwelt auf kreative Praktiken und mögliche Interaktionen wirken (Molotch 2002). Charles Landry (2000) betont in seiner Studie zur kreativen Stadt die Wirkung von Authentizität auf schöpferische Praxis. Orte stellen in ihrer einmaligen physischen und kognitiven Gestalt demnach kulturelle und symbolische Ressourcen zur Verfügung, die kreativ genutzt werden können. Infrastrukturen stiften auf diese Weise Identität und schaffen Ortsbezogenheit, die Vertrautheit und Sicherheit im kreativen Handeln schafft.

Kreatives Schaffen, Üben, Aufführen, Ausstellen und Zusammenarbeiten stellt Anforderungen an materielle Infrastrukturen und Formen von Architektur (Shaw 2013). Untersuchungen zu Eigenschaften und Qualitäten von Orten, an denen bildende und darstellende Künstler arbeiten, heben auf den häufig großen Flächenbedarf der Gestaltung und Aufführung von Kunstwerken ab. Diesen Anforderungen werden insbesondere leer stehende Räume gerecht, die für Wieder- oder Umnutzungen zur Verfügung stehen. Häu- fig sind dies Verkehrs- und Industrieinfrastrukturen, die im Zuge der De-Industrialisierung brach gefallen sind und neu konfiguriert werden können. Sie bieten temporär oder auf Dauer offene und flexible Orte für künstlerische und kreative Produktion. Eine weitere herausragende Eigenschaft dieser Räume ist die Möglichkeit zur vielfältigen Nutzung für so unterschiedliche Zwecke wie zum Beispiel Musikproben, Ausstellungen, Theateraufführungen, Cafés oder Co-Working ${ }^{1}$. Materielle Charakteristika wie Größe, Ausstattung und Multifunktionalität der Nutzung erlauben notwendige Frei- und Spielräume für kreative Experimente. Insbesondere Künstler schätzen wenig formalisierte, hierarchiearme und wenig regulierte Räume, die sie inspirieren (Drake 2003) und in denen sie ,einfach ,sein““ können (Rantisi/Leslie 2010: 2838, eigene Übersetzung).

Unter Bezugnahme auf eine Wiederbetonung des Materiellen in der Humangeographie (Jackson 2000) argumentieren Rantisi und Leslie (2010), dass materielle Faktoren und Qualitäten kreative Dispositionen durch physische und sinnesbezogene Einflüsse fördern können. Die Ausstattung mit Infrastrukturen prägt den Charakter von Stadtteilen und Nachbarschaften und ist zugleich Ergebnis sozialer Konstruktionen von Orten, deren subjektive Bedeutungen sich in Raum und Zeit verändern (Watson/Hoyler/Mager 2009). Architektonische Gestaltung und Atmosphären materieller Infrastrukturen üben darüber hinaus sensorische und affektive Einflüsse aus, die auf komplexe Weise mit Kreativitätsprozessen rückkoppeln (Gibson 2005). Entsprechend offene Räume lassen mehr oder weniger zufällige Sozialkontakte und Kommunikationsgelegenheiten zu, die zwischen kreativer Produktion und Konsumtion vermitteln. Für Jugendliche beispielsweise bieten diese Räume erste Möglichkeiten zum Einüben, Erproben und Aufführen eigener populärkultureller Praktiken vor einem kleinen Publikum (Hoyler/Mager 2005). Für sie eröffnen Freiräume außerhalb der direkten Kontrolle von Elternhaus und der pädagogischen Ansprüche der Schule Bühnen für Ideengenerierung und risikoarmes Experimentieren. Dieses Verständnis folgt einer kontextspezifischen Lesart der Konstruktion und Performanz von Kreativität in kulturellen Einrichtungen, die als ständig entstehende Orte prozessual und relational verstanden werden.

\subsection{Kreativität jenseits städtischer Kontexte}

Bislang bestimmt die Literatur zur Kultur- und Kreativwirtschaft vornehmlich Städte als den geographischen Kontext von Kreativität. Die räumliche Einbettung kreativen Handelns in urbanen Umwelten bietet eine besondere Intensität und spezifische Qualität der Verbindung von Kultur und

\footnotetext{
${ }^{1}$ Stationäre Bürogemeinschaft zur Nutzung für flexibles, überwiegend digitales Arbeiten.
} 
Ökonomie an (Scott 2000). Ein gewisses Maß an Zentralität und die Offenheit des gesellschaftlichen Klimas sind Voraussetzungen, um an Orten soziale Vernetzung, Wissenstransfer, eine ausreichende institutionelle Einbettung und den Aufbau von Reputation zu gewährleisten (Power/ Hallencreutz 2002). In ihrem Fokus auf große städtische Zentren als Orte kreativer Produktion und Konsumtion folgen auch raumplanerische Diskussionen weitgehend diesen funktional-ökonomischen Perspektiven. Kreativität, Wirtschaftsentwicklung und Stadtplanung stehen dabei in unmittelbarem Zusammenhang: Kreativität und Innovation gelten als Motor für Stadtentwicklung, Urbanität und eine erfolgreiche Entwicklungsplanung in Städten fördern Kreativität (Kirchberg 2010).

Gleichwohl bleiben empirische Studien zu Stadtentwicklung und kreativitätswirtschaftlichem Wachstum in Bezug auf kausale Zusammenhänge, sozialräumliche Auswirkungen und mögliche wirtschaftliche Effekte häufig vage (Olma 2009). Daneben blendet der rein ökonomische Fokus auf urbane Kontexte wie global cities, kulturelle Distrikte oder Szeneviertel andere Orte von Kreativität systematisch aus oder trivialisiert sie. In den vergangenen Jahren wendet sich eine wachsende Zahl empirischer Einzelstudien gegen diese Verengung der Betrachtung und verdeutlicht, dass Kreativität verschiedene formelle und informelle Ausprägungen mit großer geographischer Verbreitung annehmen kann (vgl. Gibson 2012b). Zu den fruchtbarsten Studien zählen Arbeiten zur Komplexität und Diversität von Kreativität in Kleinstädten, welche die herkömmliche Rhetorik von Vergleichen miteinander konkurrierender Städte infrage stellen und die Besonderheiten von Orten in den Blick nehmen (z. B. Jayne/Gibson/Waitt et al. 2010). Andere Arbeiten verschieben den Fokus von den städtischen Zentren an deren Randbereiche und untersuchen alltägliche Formen von Kreativität in suburbanen und peripheren Stadträumen (Waitt/Gibson 2009), die zudem Potenziale für nachhaltiges Wirtschaften besitzen können (Fleming 2009). Schließlich entdecken Studien auch auf dem Lande überraschende und innovative Projekte, deren Kreativität als Entwicklungsfaktor „durchaus eine oft unterschätzte und nicht zu vernachlässigende Rolle spielen kann“ (Ermann 2011: 27).

Grundsätzlich verdeutlichen Fallstudien, dass die strikte Zuordnung von Kreativität zu einer einzelnen Maßstabsebene die Komplexität räumlicher Verflechtungen über verschiedene Skalen hinweg verdeckt. Als Bedingung und Ergebnis sozialer Praktiken werden Kreativräume multiskalar und situativ geschaffen. Selbst gebaute kulturelle Infrastrukturen stellen in diesem Verständnis nur temporär Raum für Kreativität zur Verfügung, andere Freiräume werden spontan und ephemer auch in räumlich peripherer Lage eingerichtet, um nach nur wenigen Stunden samt kreativen Produzenten und Konsumenten wieder zu verschwinden (Ingham/Purvis/Clarke 1999). Kreativität entsteht an Orten, die durch Kontakte zu und Einflüsse von verschiedenen anderen Orten konstituiert werden und kontextspezifisch räumliche und zeitliche Maßstabsebenen miteinander verknüpfen. Diese relationale Einbettung kreativer Räume wird erst zögerlich im raumplanerischen Kontext reflektiert, wo homogenisierende Konzepte der Steuerung von Kultur- und Kreativitätswirtschaft wie zum Beispiel durch die selektive Förderung prestigeträchtiger kultureller Großprojekte schnell an ihre Grenzen zu stoßen scheinen (Evans 2009).

\subsection{Kreativität: funktionales, soziales und ermächtigendes Konzept}

Wie schwierig es ist, den Begriff Kreativität zu operationalisieren, zeigen vergleichende Studien der Kultur- und Kreativwirtschaft. So sieht beispielsweise eine deutsche Studie in Kongruenz zu Floridas Arbeiten Kreativität als ,eine Fähigkeit, die allen Menschen innewohnt ... und die sich auf vielerlei Weise in zahlreichen Tätigkeitsbereichen ausdrücken kann“ (Fritsch/Stützer 2007: 16). Die daran anschließende empirische Konzeptionalisierung des Begriffs beschränkt sich allerdings forschungspragmatisch auf die Abgrenzung entlang statistischer Dimensionen von Beschäftigung und Wirtschaftsbereichen. Wie in anderen Beispielen hat dies methodische und konzeptionelle Konsequenzen. Die definitorische Breite des Begriffs wird auf Formen von Kreativität reduziert, die in gängigen Kategorien beruflicher Beschäftigung verhaftet bleiben. Die ökonomische Logik reduziert die mögliche Vielfalt kreativer Praktiken auf sogenannte höherwertige und verwertungsbezogene Tätigkeiten der Wertschöpfung (Markusen 2006). Hinter dem Konzept ökonomischer Kreativität verbergen sich normative Vorstellungen darüber, was eine kreative Leistung ist, wer kreativ sein kann und woher kreative Menschen zu rekrutieren sind. Diese Annahmen tendieren dazu, ästhetische und funktionale Anschauungen zu bevorzugen, die entlang bestehender schichtbezogener Distinktionslinien von Wert, Leistung und Geschmack perpetuiert werden (Peck 2005).

Mittlerweile greifen Diskussionen alternative Versionen von Kreativität jenseits der dominanten Grenzziehungen und ergebnis- und produktorientierten Einteilungen auf und verweisen auf Praktiken, die für Menschen außerhalb beruflicher Kontexte und kommerzieller Motivationen Bedeutung besitzen (Brennan-Horley/Gibson 2009: 2611). Diese Vorstellungen setzen dem Elitären und Außergewöhnlichen das Banale und Gewöhnliche von Kreativität entgegen. Sie sprechen Formen alltäglicher und populärer Kultur wie beispielsweise Graffiti, dem Gärtnern auf marginalen städtischen Arealen oder dem täglichen Zubereiten von Speisen ein hohes Maß an Kreativität zu (Gibson/Klocker 2005: 100). Mit solchen vordergründig zweckfreien und unproduktiven Tätigkeiten rücken Sozialräume in den Fokus, die einen Gegenpol zu den herausgehobenen Orten 
ökonomischer Wertschöpfung darstellen. Handwerkliche, sozial betreuende, freizeitbezogene, ehrenamtliche und andere vermeintlich simple Formen von Kreativität zeigen eine andere Geographie profaner und wertvoller Praktiken (Edensor/Leslie/Millington et al. 2010).

Kreativität ist selten ausschließlich von Überlegungen zu Karrierebiographien bestimmt, sondern durch den Wunsch, sich persönlich zu engagieren und sich auf affektive Dimensionen zum Beispiel von Musik, Literatur oder Bildender Kunst einzulassen. Das Selbstverständnis der Akteure speist sich häufig weniger aus dem Zwang, verwertbare Produkte herzustellen, als vielmehr aus Spaß an der Sache oder aus Empathie und Zuneigung gegenüber anderen Menschen oder Ansichten (Gibson/Kong 2005: 544). Kreativität ist in diesem Verständnis nicht primär eine Eigenschaft von Produkten, Berufen oder Branchen, sondern das prozessuale Ergebnis individueller und kollektiver Bewertungen von Räumen und Handlungen in unterschiedlichen Kontexten. Sie ist nicht ausschließlich ökonomische Notwendigkeit und berufliche Verpflichtung, sondern stellt eine Möglichkeit zur Selbstvergewisserung und zur (gegen)kulturellen Ermächtigung innerhalb und außerhalb von Erwerbsarbeit dar (Ettlinger 2010).

\subsection{Kreativität in historischer Perspektive}

Die Anerkennung des relationalen und prozessualen Charakters von Kreativität erfordert, unabhängig von dessen formeller oder informeller Ausprägung, eine Sensibilität gegenüber spezifischen Kontexten ihrer Ausübung (Pratt 2011). Berücksichtigen Einzelstudien zur Kreativ- und Kulturökonomie mittlerweile mehr und mehr regionale Besonderheiten materieller Infrastrukturen sowie die soziale Einbettung kreativer Tätigkeiten, findet eine historische Einordnung von Kreativität selten statt. Eine Ausnahme bildet beispielsweise die breit angelegte Vergleichsstudie von Evans (2001), der für verschiedene Städte zeigt, dass heutige kulturelle Distrikte singuläre Geschichten aufweisen und auf spezifischen historischen Bedingungen von Planung, Kontrolle und Macht fußen. Er plädiert grundsätzlich für eine historische Perspektive der Langfristigkeit, welche die Entwicklungen von Kreativität pfadabhängig fasst und die komplexe Interdependenzen berücksichtigt.

Kultur- und Kreativwirtschaft gilt als vergleichsweise junges Forschungsfeld, das erst unter spätmodernen und postindustriellen Bedingungen entstehen konnte. Historische Arbeiten kritisieren diesen Aktualitätsbezug, der zwar wichtige Beiträge zum Verständnis wirtschaftlicher Dynamik und Grundlagen für räumliche Planung liefert, aber insbesondere zwei Perspektiven außer Acht lässt (vgl. Heßler/Zimmermann 2008). Zum einen erhellt die Frage nach Kontinuitäten und Brüchen der wirtschaftlichen Entwicklung Zusammenhänge von Ort, Wissen und Kreativi- tät. Hier erst werden die historischen und geographischen Potenziale lokaler Kontexte sichtbar, die entscheidende Impulse, Ressourcen und Infrastrukturen für Kreativität liefern. Historische Perspektiven verdeutlichen die Vielfalt kreativer Tätigkeiten in ihrer Abhängigkeit von institutionellen und individuellen Förderern wie Staat, Kirche und Mäzenen. Sie sensibilisieren damit für aktuelle Konstellationen der Kultur- und Kreativwirtschaft, die sich aus Akteuren des privatwirtschaftlichen, des öffentlich finanzierten und des zivilgesellschaftlichen Sektors zusammensetzen. Zum anderen schärft eine historische Perspektive den Blick für die diskursive Verfasstheit von Begriffen und ermöglicht eine Interpretation des Kreativitätsbegriffes entlang sozial-, kultur- und wirtschaftspolitischer Entwicklungslinien (Ingold/Hallam 2007). Reckwitz (2013) interpretiert den Bedeutungswandel von Kreativität von einem individuellen Modell der Selbstentfaltung hin zu einer allgegenwärtigen und verpflichtenden Kategorie der Wirtschafts- und Berufswelt vor dem Hintergrund gesellschaftlicher Modernisierung. Für ihn vollzog sich dieser Wandel aufgrund der Rationalisierung von Arbeits- und Lebensweisen parallel zu einer Ökonomisierung und Medialisierung des Sozialen. Die Lebensführung orientiert sich heute weniger an materieller als an affektiver Befriedigung, die sich in einem Bedürfnis nach Enthusiasmus und nach produktiver und konsumtiver Ästhetisierung des ständig Neuen äußert. Kreativität wird nun zugleich zum Wunsch und zur Verpflichtung.

Diese Diskussionen zeigen, dass ein rein ökonomischer Fokus auf Kultur und Kreativität dazu tendiert, aktualitätsbezogen, funktional, auf Verwertung bezogen und sozialräumlich selektiv zu argumentieren. Am Beispiel alternativer kultureller Einrichtungen und ihrer Trägergruppen in Deutschland wird Kreativität im Folgenden kontextbezogen betrachtet. Ziel ist es, die Vielfalt alltäglicher Kreativität in die sozial- und kulturpolitischen Diskussionen der vergangenen Jahrzehnte einzuordnen, um konkrete Praktiken des Umgangs mit kulturellen Infrastrukturen in verschiedenen geographischen und historischen Kontexten sichtbar zu machen.

\section{Kultur und Kreativität im Kontext: zur Geschichte soziokultureller Initiativen und Einrichtungen}

Kulturpolitische Diskussionen und die damit verbundenen Entwicklungen kultureller Einrichtungen lassen sich als Reaktion auf Wünsche, Herausforderungen und Krisenphänomene der Gesellschaft interpretieren. In Deutschland können für die Zeit nach dem Zweiten Weltkrieg verschiedene Phasen der Kulturpolitik analytisch unterschieden werden, die von spezifischen sozialen Gruppen oder ,Generationen ${ }^{\circ}$ getragen wurden (Göschel/Mittag/Strittmatter 1995). Von besonderer Bedeutung ist dabei die Phase des sogenannten 
Kulturkonflikts von der Mitte der 1960er bis in die 1980er Jahre, in der eine reformierte Neue Kulturpolitik auf Brüche und Konfliktlinien innerhalb der Gesellschaft reagierte.

Insgesamt lassen sich drei krisenhafte Erscheinungen als Auslöser dieser Diskussionen identifizieren. Erstens gerieten der tradierte Kulturbegriff und seine Funktionalisierung in die Kritik. Ausgehend unter anderem von der ideologieund herrschaftskritischen Haltung der Frankfurter Schule wurde die normative Bedeutungsdimension des Kulturbegriffs zunehmend infrage gestellt (Marcuse 1965). Die Vorstellung von Hochkultur als das Wahre, Schöne und Gute maskiere, so die Argumentation, ihre tatsächlichen sozialen, ökonomischen und politischen Funktionen. Diese normatividealisierende Betrachtung von Hochkultur werte alltägliche Praktiken der Lebensgestaltung ab. Ein erweitertes Verständnis sprach Kultur unter Einbezug aller menschlichen Lebensbereiche eine potenziell emanzipative und demokratisierende Rolle zu. Kultureinrichtungen sollten sich inhaltlich gegenüber Alltagsthemen öffnen und Bürger unter den Schlagworten „Kultur für alle“ und „Kultur von allen“ zu Kommunikation, Partizipation und kreativer Auseinandersetzung mit sich selbst und mit ihrer Umwelt animieren (Glaser/Stahl 1983). Zweitens zeichnete sich ein Anhalten der Bildungskrise $a b$, die sich trotz der Bemühungen um sozialstaatliche Umverteilung mit dem Ziel der Chancengleichheit nicht entschärfte. Eine neue Zielsetzung von Kulturpolitik sollte sein, Lernprozesse, die soziale und politische Bedingungen zum Gegenstand bewusster und gezielter Veränderungen machen, auch außerhalb des Schulsystems $\mathrm{zu}$ initiieren. Kultureinrichtungen wurden auf diese Weise als Bildungseinrichtungen interpretiert und den gleichen Demokratisierungs- und Gleichheitsprinzipien unterworfen. Ein dritter auslösender Faktor der Neuen Kulturpolitik war eine seit den 1960er Jahren konstatierte Krise der Stadt. Die funktionale Entmischung der ehemals hochgradig integrierten Stadt führe zu „Unwirtlichkeit“ und ziehe soziale Entfremdung, Anonymität und Apathie nach sich (Mitscherlich 1965). Bildung, Kunst und Kultur müssten gegen diese planerisch, technisch und ökonomisch bestimmten Deformationen aktiviert werden. Kulturelle Einrichtungen sollten „Spiel-“ und „Freiräume“ eröffnen, kreativitätsfördernde Kommunikationsprozesse anstoßen und damit der Verödung der Städte entgegenwirken (Glaser/Stahl 1983).

Die Reaktionen auf diese Krisenerscheinungen waren zunächst getragen von traditionellen sozialen Bewegungen, die soziale Auseinandersetzungen primär als Verteilungskonflikte des gesellschaftlichen Reichtums interpretierten. Daneben existierten Ideen, die Selbstverwirklichung und kulturelle Teilhabe an kreativer Produktion und Konsumtion als ein zentrales Bürgerrecht ansahen (Sievers/Wagner 1992). Neu traten während der 1970er Jahre Kräfte hinzu, die sich ausdrücklich gegen die kulturelle und ökonomische Dominanz hegemonialer Gruppen aussprachen und als Sub- und Gegenkulturen grundsätzliche Opposition artikulierten. Als bedeutendste Träger der Neuen Kulturpolitik dominierten seit den späten 1970er Jahre Gruppen, die sich auf situative Beeinträchtigungen und Bedrohungen etwa durch Sanierungsmaßnahmen, Umweltverschmutzungen und Großprojekte der Verkehrsplanung oder atomaren Energiegewinnung bezogen. Diese neuen sozialen Bewegungen reagierten auf einen drohenden Identitätsverlust, hervorgerufen durch unüberschaubare Systeme wie Markt, Medien und Bürokratie. Kreative Selbstverwirklichung und Authentizität waren Ziele, die unter dem identitätsstiftenden Bezug zur Gemeinde, zum Stadtteil oder zum Quartier möglichst alle Beteiligten einbeziehen wollten (Rammstedt/ Wagner 1998).

Abgeleitet aus den Grundsätzen der Demokratisierung von Kultur, den Potenzialen kommunikativer Alltagsbezüge und den emanzipativen Prozessen kreativer Selbstentfaltung bestand die Aufgabe der Neuen Kulturpolitik darin, einerseits die traditionellen Kultureinrichtungen einem breiteren Publikum zu öffnen und andererseits neue Möglichkeiten für kreative Praktiken zu schaffen. Ein Ergebnis dieser Bemühungen war die Etablierung neuer Einrichtungen wie Kommunikationszentren, Bürgerhäuser oder Kulturläden, die „ökologische Nischen“ und „sozio-kulturelle Lebensräume" für die Kreativität der Nutzer bieten sollten. In vielen deutschen Kommunen gestalteten Initiativen Bahnhöfe, Zechen oder Lagerhallen in Eigenregie zu Kulturräumen um (vgl. Hübner 1981; Mager 2000). Nachdem 1979 die „Bundesvereinigung sozio-kultureller Zentren“ als Verein in Wilhelmshaven gegründet wurde, schlossen sich nach und nach immer mehr der neuen Kulturzentren dem zentralen Verband an, der sich in seiner Gründungssatzung folgenden Zielen der Kulturarbeit verschrieben hat: „Basisund Nutzerorientierung; Integration verschiedener Altersgruppen, sozialer Schichten und Nationalitäten; Offenheit und Transparenz; Formen sozialer politischer Arbeit sowie demokratischer Kultur (Initiierung sozialer, politischer und kultureller Lernprozesse); demokratische Entscheidungsstruktur; nicht kommerzielle Ausrichtung" (Bundesvereinigung sozio-kultureller Zentren 1977: o. S.).

Als Leitideen von Soziokultur können damit gelten: die Einbeziehung sozial und kulturell benachteiligter gesellschaftlicher Gruppen in eine ,demokratisierte Kultur', die Betonung der politischen Bezüge von Kultur in einem herrschaftskritischen Verständnis, die Selbstverwaltung und -organisation von Kultur inklusive des Aufbaus einer materiellen und sozialen Infrastruktur, die Etablierung neuer Formen und Inhalte jenseits des traditionellen Kulturbetriebs sowie die Förderung der kreativen, nicht-kommerziellen Selbstbetätigung (Glaser/Stahl 1983: 210 ff.). Mit diesem Selbstverständnis nimmt Soziokultur die Anregungen der Neuen Kulturpolitik auf und versucht, verschiedene Ansätze von Kultur-, Bildungs-, Freizeit- und Sozialpolitik in die Pra- 
xis der Kulturarbeit zu überführen. Das Spektrum der Arbeit umfasst beispielsweise kulturelle Bildung und Weiterbildung, interkulturelle Angebote, psychosoziale Arbeit, Werkstätten für bildende Kunst, Musik, Theater oder Geschichte, die Unterstützung von Selbsthilfegruppen sowie die politische Arbeit, was je nach Akzentsetzung zu sehr unterschiedlichen Umsetzungen führen kann. Konsequenterweise verstehen die Zentren ihre Besucher nicht als Kunden oder als Publikum, sondern als Nutzer, welche die Grenze kultureller Konsumtion und Produktion selbst ausloten. In jüngster Zeit erfährt die Kulturarbeit soziokultureller Einrichtungen zudem Anerkennung für ihre Verdienste um die Kultur- und Kreativwirtschaft, wenn die Zentren als Nischen für gerade noch nicht kommerzialisierte Subkulturen, als Ausbildungs- und Qualifizierungsorte für Kreative, als Markteinstiegsorte für junge Künstler, als Schnittstellen und Vernetzungsknoten mit Kreativunternehmen oder als Sammelorte von Zeitspenden Ehrenamtlicher gelobt werden (Der Beauftragte der Bundesregierung für Kultur und Medien 2012).

Diese Entwicklungen sind kein rein westdeutsches Phänomen. Die Ursprünge der Begriffe Soziokultur und soziokulturelles Zentrum liegen in westeuropäischen kulturpolitischen Debatten des Rats für kulturelle Zusammenarbeit des Europarats in den 1960er und 1970er Jahren. Für viele Länder Europas sind Kommunikationshäuser und Sozialzentren dokumentiert, die einem soziokulturellen Ansatz folgen (vgl. Evans 2001). In Ostdeutschland bot erst die Wiedervereinigung die Möglichkeit, eine entsprechende Infrastruktur aufzubauen. Zwar existierten soziale und kulturelle Strömungen, die sich ähnlich wie in Westdeutschland gegen herrschende politische Strukturen und für eine selbstbestimmtere Ausübung von Kultur einsetzten. Die Dominanz und Kontrolle der staatlichen und gewerkschaftlichen Kultureinrichtungen allerdings verhinderte weitgehend den Aufbau unabhängiger Strukturen, welche die wenigen privaten und kirchlichen Freiräume hätten verstetigen können. Mit der deutschen Einigung wurde der Begriff Soziokultur auch in Ostdeutschland eingeführt, zunächst um eine Vielzahl kultureller Einrichtungen hauptsächlich begrifflich zu bündeln und finanzierungspolitisch zu verankern. Einerseits wurden die vorhandenen breitenkulturellen Infrastruktureinrichtungen in neue Trägerschaften überführt und umstrukturiert, andererseits entstanden völlig neue Einrichtungen aus künstlerischen und kulturellen Initiativen (vgl. Groschopp 2001).

\section{Standorte und Strukturen soziokultureller Zentren und Initiativen in deutschen Städten und Gemeinden}

Seit 1992 erhebt die „Bundesvereinigung soziokultureller Zentren e. V." mit Sitz in Berlin in unregelmäßigen Abständen Daten ihrer Mitgliedseinrichtungen, die in ers- ter Linie dazu dienen, Rahmenbedingungen und Leistungen soziokultureller Arbeit zu identifizieren, zu bewerten und Handlungsoptionen abzuleiten. Informationen zu den Schwerpunkten der Kulturarbeit, der Situation von Nutzern, Engagierten und Beschäftigten sowie den finanziellen und materiellen Rahmenbedingungen sind außerdem Grundlage für die Außendarstellung gegenüber der Politik und Öffentlichkeit sowie der Formulierung von Forderungen gegenüber Kommunen, den Ländern und dem Bund. Die Vereinigung sieht ihre Hauptaufgaben in der Interessenvertretung ihrer Mitglieder auf unterschiedlichen politischen Ebenen, der Erschließung und Verteilung von Informationen und Ressourcen, der Personalentwicklung und Beratung der Mitgliedseinrichtungen sowie in der Öffentlichkeitsarbeit (Bundesvereinigung soziokultureller Zentren 2012). Ähnlich anderer Bundeskulturverbände wie beispielsweise der „Deutsche Bühnenverein“ oder der „Bundesverband Bildender Künstlerinnen und Künstler" ist die Vereinigung Mitglied im Deutschen Kulturrat und damit an bundesweit spartenübergreifenden kulturpolitischen Diskussionen beteiligt. 2012 waren insgesamt 474 Einrichtungen und Initiativen aus 14 Landesverbänden in der Bundesvereinigung organisiert, hinzu kommen 50 Einrichtungen im selbstständigen Landesverband „Soziokultur Sachsen“. Das Motto der Bundesvereinigung „Vielfalt. Aus Prinzip.“ weist auf die unterschiedlichen Organisationsformen der Zentren und Initiativen hin, die von kleinen, nur durch ehrenamtliche Tätigkeit getragenen Vereinen ohne eigene Räumlichkeiten bis hin zu Großzentren mit über zehn hauptamtlichen Mitarbeitern, einer infrastrukturellen Ausstattung von Büro- und Veranstaltungsräumen mit mehreren Hundert Quadratmetern Nutzfläche und einem breit gefächerten Kulturangebot reichen. Überregional bekannte Beispiele sind die Zentren „Lagerhalle“ in Osnabrück, „Pavillon“ in Hannover, „Tollhaus“ in Karlsruhe oder „Zeche Carl“ in Essen.

Das hier vorgestellte empirische Material stammt aus den beiden jüngsten Befragungen aller Mitgliedseinrichtungen, die in den Jahren 2010 und 2012 mithilfe eines OnlineFragebogens durchgeführt wurden. Der Rücklauf betrug 52,8\% (2010) beziehungsweise 54\% (2012). Um Aussagen über alle Mitgliedseinrichtungen treffen zu können, wurden die erzielten Befragungsergebnisse, wo möglich, mithilfe eines Gewichtungsfaktors hochgerechnet, dem die Einnahmesituation der Zentren (Projekt-, Investitions- und Personalförderung, Eigenerwirtschaftung, Mitgliedsbeiträge und Spenden) im Jahr 2011 zugrunde liegt. Fehlende Angaben $\mathrm{zu}$ Alter und Standort von Einrichtungen wurden nachrecherchiert. Jährlich erreichen die in der Vereinigung organisierten soziokulturellen Zentren bundesweit mit knapp 90.000 offenen Treffs, Workshops, Kursen und Veranstaltungen rund 9,5 Mio Nutzer. Knapp $60 \%$ der rund 24.000 in soziokulturellen Zentren Engagierten sind Ehrenamtliche (Ahbe/Dallmann/Mager et al. 2013: 9 ff.). 


\subsection{Diffusion}

Aus geographischer Perspektive kann die Ausbreitung der Innovation „soziokulturelles Zentrum“ zwischen 1962 und 2010 als Diffusionsprozess gekennzeichnet werden, der bis Ende der 1970er Jahre durch ein nur langsames, anschlieBend durch ein beschleunigtes, beinahe lineares und $a b$ etwa 2000 wiederum durch ein langsameres Wachstum charakterisiert ist. In den 1980er und 1990er Jahren werden zwei Drittel aller soziokulturellen Einrichtungen gegründet, danach nimmt die Zahl neuer Gründungen $a b$ und erreicht lediglich das Niveau der 1970er Jahre (vgl. Tab. 1). Während die meisten Zentrumsgründungen in Westdeutschland in die Phase der Neuen Kulturpolitik in den 1980er Jahren fallen und die Verlangsamung des Wachstums bereits im Folgejahrzehnt einsetzt, kann die Entwicklung in Ostdeutschland nach 1990 als Aufsetzen eines zweiten Diffusionsprozesses interpretiert werden.

Die räumliche Ausbreitung soziokultureller Zentren zeigt zu Beginn einen urbanen Fokus (vgl. Abb. 1). Mitte der 1970er Jahre existieren in West-Berlin, Dortmund, Hamburg, Nürnberg und Stuttgart erste Einrichtungen ebenso wie in Göttingen, Osnabrück, Trier und einzelnen Städten im Ruhrgebiet. Zugleich entstehen ausgehend von politisch-kulturellen Clubs oder inspiriert von der Schüler- und Studentenbewegung Einrichtungen außerhalb der Verdichtungsräume etwa in Schorndorf in Baden-Württemberg, auf Burg Waldeck im Hunsrück oder in Eckernförde in Schleswig-Holstein. Mit Ausnahme von Duisburg und München eröffnet in jeder deutschen Großstadt über 500.000 Einwohner bis in die 1980er Jahre mindestens eine soziokulturelle Einrichtung. Darüber hinaus kommt es in Städten mit mehrheitlich sozialdemokratischer Kommunalregie- rung wie Hamburg, Bremen und Nürnberg zum Ausbau der stadtteilbezogenen Infrastruktur, der zu großen Teilen von soziokulturellen Einrichtungen getragen wird. Bemerkenswert ist die Konzentration von Neugründungen in der Region Stuttgart. In die 1980er Jahre fällt auch die Gründung von Zentren in kleineren Großstädten, insbesondere wenn sie zugleich Universitätsstandorte sind wie beispielsweise Bonn, Freiburg, Heidelberg, Marburg oder Tübingen. In den Bundesländern Baden-Württemberg, Niedersachsen und Nordrhein-Westfalen zeichnet sich eine flächenhafte Erschließung ab. Nach der Deutschen Wiedervereinigung wird der Zuwachs zunächst vor allem von Einrichtungen in Ostdeutschland getragen, deren Zahl am stärksten in Thüringen und Sachsen wächst. Neben Neugründungen finden sich dort auch soziokulturelle Zentren, deren dezentraler Charakter auf die Reorganisation bestehender staatlicher Infrastruktur zurückzuführen ist. $\mathrm{Zu}$ einem Ausbau kommt es vor allem im Ruhrgebiet und Südniedersachsen, nur zaghaft verdichtet sich die vergleichsweise geringe Ausstattung mit soziokultureller Infrastruktur in Bayern und Rheinland-Pfalz. Im vergangenen Jahrzehnt entstehen nur vereinzelt neue soziokulturelle Initiativen, nicht selten in Städten und Gemeinden, die bereits Standorte einer anderen Einrichtung sind, oder als neue und kleine Ankerpunkte in verhältnismäßig peripheren Gebieten wie etwa in Mecklenburg-Vorpommern.

\subsection{Markant in Stadt und Land}

Auf der geographischen Makroebene zeigen die Standortmuster eine Orientierung am hierarchischen Siedlungssystem. Soziokulturelle Einrichtungen sind damit, den tradierten Kultureinrichtungen nicht unähnlich, eher in

Tab. 1 Alters- und Standortstruktur soziokultureller Zentren. (Quellen: Bundesvereinigung soziokultureller Zentren; Bundesinstitut für Bau-, Stadt- und Raumforschung; eigene Recherchen und Berechnungen)

\begin{tabular}{|c|c|c|c|c|c|c|c|}
\hline \multirow[t]{2}{*}{ Kreistyp 2008 (zusammengefasst) } & & \multicolumn{5}{|c|}{ Gründungsjahr } & \multirow[t]{2}{*}{ GESAMT } \\
\hline & & $1962-1969$ & 1970-1979 & 1980-1989 & 1990-1999 & $2000-2009$ & \\
\hline \multirow[t]{3}{*}{ Kernstädte } & Absolut & 7 & 35 & 65 & 54 & 27 & 188 \\
\hline & Spaltenanteil & $58,3 \%$ & $64,8 \%$ & $52,4 \%$ & $35,3 \%$ & $40,3 \%$ & $45,9 \%$ \\
\hline & Zeilenanteil & $3,7 \%$ & $18,6 \%$ & $34,6 \%$ & $28,7 \%$ & $14,4 \%$ & \\
\hline \multirow[t]{3}{*}{ Verdichtetes Umland } & Absolut & 3 & 12 & 41 & 47 & 20 & 123 \\
\hline & Spaltenanteil & $25,0 \%$ & $22,2 \%$ & $33,1 \%$ & $30,7 \%$ & $29,9 \%$ & $30,0 \%$ \\
\hline & Zeilenanteil & $2,4 \%$ & $9,8 \%$ & $33,3 \%$ & $38,2 \%$ & $16,3 \%$ & \\
\hline \multirow[t]{3}{*}{ Ländliches Umland } & Absolut & 2 & 4 & 7 & 25 & 14 & 52 \\
\hline & Spaltenanteil & $16,7 \%$ & $7,4 \%$ & $5,6 \%$ & $16,3 \%$ & $20,9 \%$ & $12,7 \%$ \\
\hline & Zeilenanteil & $3,8 \%$ & $7,7 \%$ & $13,5 \%$ & $48,1 \%$ & $26,9 \%$ & \\
\hline \multirow[t]{3}{*}{ Ländlicher Raum } & Absolut & $\mathbf{0}$ & 3 & 11 & 27 & 6 & 47 \\
\hline & Spaltenanteil & $0,0 \%$ & $5,6 \%$ & $8,9 \%$ & $17,6 \%$ & $9,0 \%$ & $11,5 \%$ \\
\hline & Zeilenanteil & $0,0 \%$ & $6,4 \%$ & $23,4 \%$ & $57,4 \%$ & $12,8 \%$ & \\
\hline \multirow[t]{3}{*}{ GESAMT } & Absolut & 12 & 54 & 124 & 153 & 67 & 410 \\
\hline & Spaltenanteil & & & & & & $100,0 \%$ \\
\hline & Zeilenanteil & $2,9 \%$ & $13,2 \%$ & $30,2 \%$ & $37,3 \%$ & $16,3 \%$ & $100,0 \%$ \\
\hline
\end{tabular}




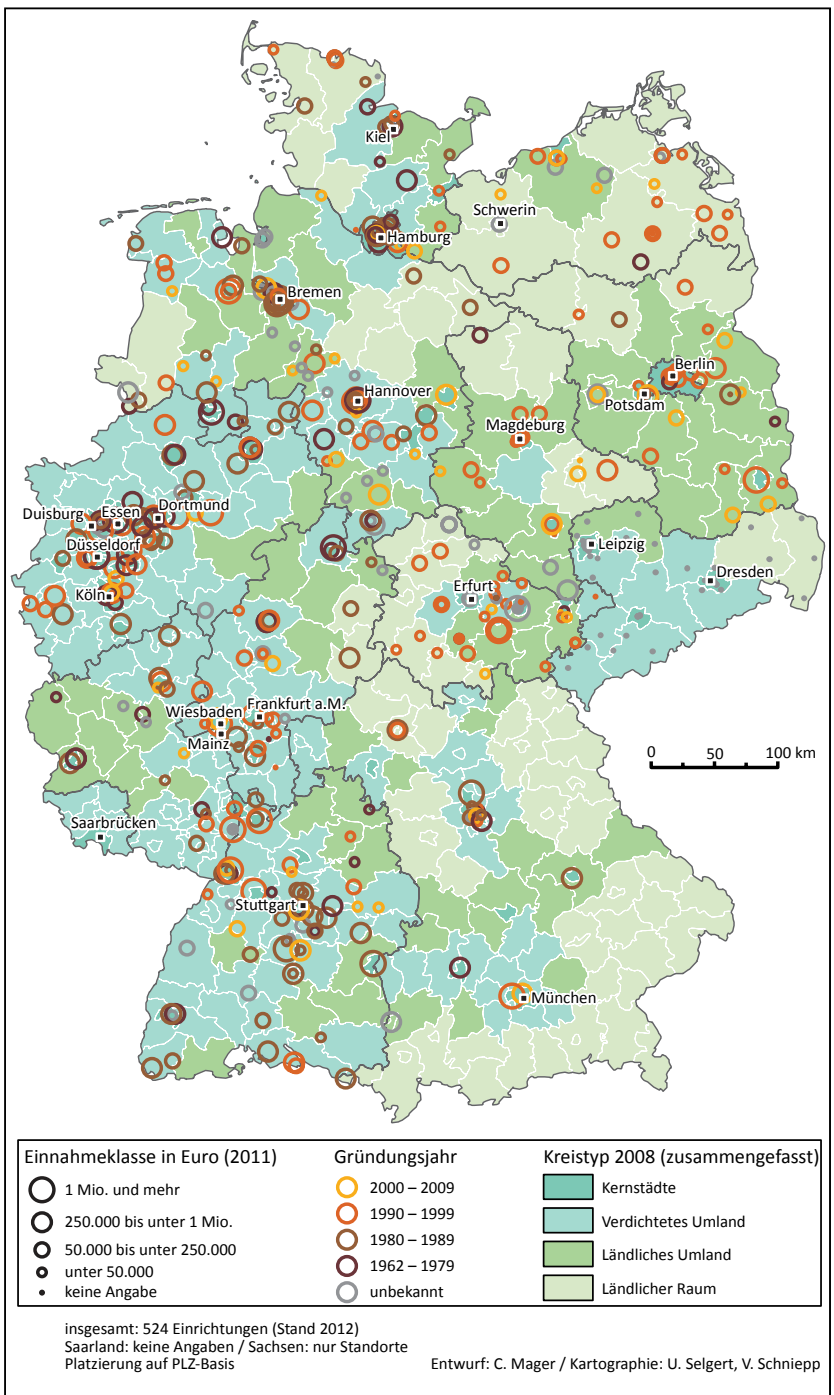

Abb. 1 Soziokulturelle Zentren 2012. (Quellen: Bundesvereinigung soziokultureller Zentren; Landesverband Soziokultur Sachsen; Bundesinstitut für Bau-, Stadt- und Raumforschung; eigene Recherchen)

Orten einer höheren zentralörtlichen Stufe zu finden. Mittelund Großstädte sind deutlich besser versorgt als Kleinstädte und kleinere Gemeinden, obgleich die Stadt-Land-Kontraste der Versorgung mit soziokulturellen Einrichtungen weniger deutlich ausfallen als jene mit traditionellen Kultureinrichtungen (vgl. Freytag/Hoyler/Mager 2002). Für 2010 beispielsweise liegen die Versorgungsgrade mit soziokulturellen Einrichtungen in Gemeinden mit 20.000 bis unter 200.000 Einwohnern deutlich über denen mit öffentlich geförderten Bühnen. Verfügt in der genannten Größenklasse nur eine von neun Kommunen über mindestens ein Theater, sind es im Falle soziokultureller Einrichtungen mehr als ein Fünftel der 655 Gemeinden.

Tabelle 1 schlüsselt die Alters- und Standortstruktur soziokultureller Zentren nach Raumordnungskategorien auf. Im vorliegenden Fall dienen zusammengefasste Kreis- typen als regionstypübergreifendes Differenzierungskriterium, um einerseits eine hinreichende Differenzierung der siedlungsstrukturellen Entwicklung nach ländlichen beziehungsweise städtischen Regionen zu ermöglichen und andererseits die Bedeutung von Stadt-Umland-Beziehungen für die Einzugsbereiche kultureller Infrastrukturen zu berücksichtigen. Insgesamt befinden sich in Deutschland etwa drei Viertel aller soziokulturellen Zentren in Kernstädten und ihrem verdichteten Umland, das restliche Viertel im ländlichen Raum im weiteren Sinne (Kategorien ländliches Umland und ländlicher Raum). Im Zeitverlauf zeigt sich, dass in den ersten drei Jahrzehnten der Schwerpunkt der Aktivität in den Kernstädten liegt, wo deutlich mehr als die Hälfte aller Einrichtungen etabliert wird. Hier und im verdichteten Umland liegen vier von fünf der bis 1990 gegründeten Zentren. In den vergangenen beiden Jahrzehnten allerdings hat sich das Verhältnis zugunsten der ländlichen Gebiete verschoben, als rund 30\% der neuen Einrichtungen dort gegründet wurden. Allein in den 1990er Jahren sind mehr als die Hälfte aller heutigen Einrichtungen im ländlichen Umland und im ländlichen Raum entstanden.

Dies ist zum einen auf die Standorttradition in Ostdeutschland zurückzuführen, wo vor 1990 der dezentralen Versorgung der Bevölkerung in Klubs und Kultureinrichtungen der verstaatlichten Betriebe und SED-nahen Massenorganisationen eine herausragende Bedeutung zukam (Strittmatter 1992). Zum anderen legen die Zuwachsraten im vergangenen Jahrzehnt Interpretationen nahe, nach denen die Bedeutung von Kultur auf dem Lande einem Wandel unterworfen ist. So wirken sich Wanderungsprozesse auf interregionaler und regionaler Ebene auf die soziodemographische Zusammensetzung der ländlichen Bevölkerung aus, was in Befunden einer Enttraditionalisierung der Sozialbeziehungen und des alltäglichen Lebens zum Ausdruck kommt. Die tendenzielle Schwächung schichtspezifischer Kategorien zugunsten ästhetisch-expressiver Lebensstilorientierungen als Erklärungsmuster für Einstellungen und Praktiken korrespondiert mit dem wachsenden Bedürfnis, auch auf dem Lande offene Formen kultureller und kreativer Vergesellschaftung zu suchen (Ermann 2011). Dem Mangel an nennenswerter öffentlicher kultureller Infrastruktur im ländlichen Raum wird vielerorts durch die Etablierung kultureller und künstlerischer Alternativen begegnet, die in der Regel zivilgesellschaftlichen Charakter tragen. Einrichtungen wie soziokulturelle Zentren sind häufig die einzigen Ankerpunkte kultureller Kreativität für ein lokales und überlokales ländliches Einzugsgebiet. Diese Ergebnisse decken sich mit Befunden, nach denen die Bedeutung des intermediären Sektors mit abnehmender zentralörtlicher Hierarchiestufe relativ ansteigt (Ebert 2008: 301). Soziokultur im ländlichen Raum kann neben ,sinnstiftenden, identitäts- und kreativitätsfördernden Leistungen ... wertvolle Beiträge zur Regionalentwicklung leisten: Erhalt von Öffentlichkeit und 
Kommunikationsgelegenheiten, Gegensteuern zur Abwanderung der Jugend, Integration von Senioren, Ausländern oder Aussiedlern und letztlich Erhöhung der Lebensqualität“" (Dallmann 2008: 17).

\section{Fazit}

Soziokultur fördert Ideen und Ausprägungen von Kreativität, die in einem spezifischen raum-zeitlichen Kontext entstanden sind. Verschiedene Krisenerscheinungen der Industriegesellschaft befeuerten das Bedürfnis, Kultur als Gut ,für alle' zu denken und die Herstellung dieses Gutes ,allen' zuzutrauen. Dieses Programm der Demokratisierung erforderte von der Neuen Kulturpolitik einen Modus von Kulturproduktion und Kulturkonsumtion, der Amateure, das Laientum und eine Do-it-yourself-Haltung gleichberechtigt neben den professionellen Kulturbetrieb und die Kulturökonomie stellt. Kreativ sein heißt dabei weniger, marktförmige Produkte und Innovationen oder verwertbares Wissen hervorzubringen, sondern in alltagsweltlichen Kontexten als Subjekt selbstbewusst und kritisch zu bestehen. Zur Umsetzung der Grundidee, die „Vergesellschaftung [der Kulturpolitik] von unten“" (von Beyme 2012: 170) materiell wie ideell zu tragen und Personen unterschiedlicher ethnischer, sozialer und räumlicher Herkunft Möglichkeiten zur selbstgesteuerten Kreativität zu bieten, bedarf es Infrastrukturen, welche die Aufgabe haben, multifunktionale Freiräume und Spielräume wohnraumnah, möglichst kostenfrei und ,niedrigschwellig' zugänglich zu machen. Soziokulturelle Zentren machen sich diese Aufgabe zu eigen und bieten Räume, Zeitressourcen und technisch-organisatorische Unterstützung für die Durchführung kultureller Projekte und kreativer Betätigung mit großer geographischer Verbreitung und Reichweite. Mittlerweile öffnen sich auch etablierte Kultureinrichtungen solchen partizipativen und inkludierenden Wegen der Kulturarbeit, und auch neue soziale Orte - beispielsweise das Co-Working - ermöglichen Austausch und Experiment mit ökonomisch-kultureller Kreativität.

Soziokulturelle Zentren gelten heute als wichtiger Teil der kulturellen Infrastruktur in deutschen Städten und Gemeinden, ihr geographischer Ausbreitungsprozess kann als Erfolgsgeschichte gelesen werden. Dass die Zentren nicht frei von ökonomischen und politischen Zwängen arbeiten können, zeigt sich in der zunehmenden Konkurrenz $\mathrm{zu}$ anderen kulturellen und freizeitbezogenen Angeboten um Nutzer und um staatliche Förderung. Mit einer Unterordnung unter den kreativwirtschaftlichen Diskurs allerdings läuft Alternativkultur Gefahr, von einer Perspektive vereinnahmt zu werden, die Kreativität historisch, sozial, inhaltlich und räumlich selektiv denkt. Wenn ein Mitarbeiter eines soziokulturellen Zentrums am Ende der Mitgliederbefragung 2012 im Freitextfeld anmerkt „Warum immer diese Statistik, wofür diese Rechtfertigungspapiere[? L]asst uns auf anderer Ebene zusammen arbeiten", so verweist er auf die klassische Strategie alternativer kultureller Einrichtungen, eine Vielfalt von Orten profaner Kreativität jenseits ökonomischer Verwertungszusammenhänge zu befördern.

Danksagung Die Bundesvereinigung soziokultureller Zentren hat dankenswerterweise der Verwendung des Datenmaterials ihrer Mitgliederbefragungen 2010 und 2012 zugestimmt. Jana Langendörfer besorgte am Institut für Geographie und Geoökologie des Karlsruher Instituts für Technologie die Bereinigung der Datengrundlagen. Der Autor dankt Michael Hoyler für hilfreiche Kommentare zu einer Manuskriptfassung.

\section{Literatur}

Ahbe, E.; Dallmann, G.; Mager, C.; Staal, M.; Trimborn-Bruns, I.; Wolter, M. (2013): Soziokulturelle Zentren in Zahlen: Statistischer Bericht 2013. Berlin.

Amin, A.; Thrift, N. (2007): Cultural-economy and cities. In: Progress in Human Geography 31 (2), 143-161.

Behr, V.; Gnad, F.; Kunzmann, K. R. (Hrsg.) (1989): Kultur, Wirtschaft, Stadtentwicklung. Dortmund. = Dortmunder Beiträge zur Raumplanung 51.

Berndt, C.; Glückler, J. (Hrsg.) (2006): Denkanstöße zu einer anderen Geographie der Ökonomie. Bielefeld.

Brennan-Horley, C.; Gibson, C. (2009): Where is creativity in the city? Integrating qualitative and GIS methods. In: Environment and Planning A 41 (11), 2595-2614.

Bundesvereinigung sozio-kultureller Zentren (1977): Satzung. Mimeo. O.O.

Bundesvereinigung soziokultureller Zentren (2012): Leitbild. http:// www.soziokultur.de/bsz/sites/default/files/file/LeitbildSoziokultur.pdf (30.07.2014).

Castree, N. (2004): Economy and culture are dead! Long live economy and culture! In: Progress in Human Geography 28 (2), 204-226.

Christaller, W. (1968): Die zentralen Orte in Süddeutschland. Darmstadt.

Crang, P. (1997): Cultural turns and the (re)constitution of economic geography. In: Lee, R.; Wills, J. (Hrsg.): Geographies of economies. London, 3-15.

Dallmann, G. (2008): Soziokultur in ländlichen Räumen. In: Politik und Kultur 5, 17.

Der Beauftragte der Bundesregierung für Kultur und Medien (Hrsg.) (2012): Kultur- und Kreativwirtschaft im Rahmen des öffentlich geförderten und des intermediären Kultursektors. Berlin.

Drake, G. (2003): 'This place gives me space': place and creativity in the creative industries. In: Geoforum 34 (4), 511-524.

Ebert, R. (2008): „Kreative Räume“ der Kultur- und Kreativwirtschaft in der Hierarchie der Städte und Anforderungen an eine zukunftsorientierte Stadtpolitik. In: Institut für Kulturpolitik der Kulturpolitischen Gesellschaft (Hrsg.): Jahrbuch für Kulturpolitik 2008: Thema Kreativwirtschaft und Kreative Stadt. Essen, 293-304.

Edensor, T.; Leslie, D.; Millington, S.; Rantisi, N. M. (Hrsg.) (2010): Spaces of vernacular creativity: rethinking the cultural economy. London.

Enquete-Kommission (2007): Schlussbericht der Enquete-Kommission „Kultur in Deutschland“. Deutscher Bundestag Drucksache 16/7000. Berlin.

Ermann, U. (2011): Kunst und Kreativität als Faktor ländlicher Ökonomien: vernachlässigbar oder unterschätzt? In: Geographische Rundschau 63 (2), 20-28. 
Ettlinger, N. (2010): Bringing the everyday in the culture/creativity discourse. In: Human Geography 3 (1), 49-59.

Evans, G. (2001): Cultural planning: an urban renaissance? London.

Evans, G. (2009): Creative cities, creative spaces and urban policy. In: Urban Studies 46 (5-6), 1003-1040.

Fleming, R. C. (2009): Creative economic development, sustainability, and exclusion in rural areas. In: Geographical Review 99 (1), 61-80.

Florida, R. (2002): The rise of the creative class: and how it's transforming work, leisure, community and everyday life. New York.

Freytag, T.; Hoyler, M.; Mager, C. (2002): Soziokultur und ihre Zentren. In: Institut für Länderkunde (Hrsg.): Nationalatlas Bundesrepublik Deutschland. Bd. 6: Bildung und Kultur. Heidelberg, 118-119.

Fritsch, M.; Stützer, M. (2007): Die Geographie der Kreativen Klasse in Deutschland. In: Raumforschung und Raumordnung 65 (1), $15-29$.

Gibson, C. (2003): Cultures at work: why 'culture' matters in research on the 'cultural' industries. In: Social \& Cultural Geography 4 (2), 201-215.

Gibson, C. (2005): Recording studios: relational spaces of creativity in the city. In: Built Environment 31 (3), 192-207.

Gibson, C. (2012a): Cultural economy: achievements, divergences, future prospects. In: Geographical Research 50 (3), 282-290.

Gibson, C. (2012b): Creativity in peripheral places: redefining the creative industries. Milton.

Gibson, C.; Klocker, N. (2005): The 'cultural turn' in Australian regional economic development discourse: neoliberalising creativity? In: Geographical Research 43 (1), 93-102.

Gibson, C.; Kong, L. (2005): Cultural economy: a critical review. In: Progress in Human Geography 29 (5), 541-561.

Glaser, H.; Stahl, K. H. (1983): Bürgerrecht Kultur. Frankfurt am Main.

Glückler, J.; Ries, M.; Schmid, H. (2010): Kreative Ökonomie. Perspektiven schöpferischer Arbeit in der Stadt Heidelberg. Heidelberg. $=$ Heidelberger Geographische Arbeiten 131 .

Göschel, A.; Mittag, K.; Strittmatter, T. (1995): Die befragte Reform: Neue Kulturpolitik in Ost und West. Berlin. = Difu-Beiträge zur Stadtforschung 15.

Groschopp, H. (2001): Breitenkultur in Ostdeutschland: Herkunft und Wende - wohin? In: Aus Politik und Zeitgeschichte B 11, 15-22.

Häußermann, H.; Läpple, D.; Siebel, W. (2008): Stadtpolitik. Frankfurt am Main.

Heßler, M.; Zimmermann, C. (Hrsg.) (2008): Creative urban milieus: historical perspectives on culture, economy, and the city. Frankfurt am Main.

Hoyler, M.; Mager, C. (2005): ,HipHop ist im Haus': cultural policy, community centres, and the making of hip-hop music in Germany. In: Built Environment 31 (3), 237-254.

Hübner, I. (1981): Kulturzentren: Gesellschaftliche Ursachen, empirische Befunde, Perspektiven sozio-kultureller Zentren. Weinheim.

Ingham, J.; Purvis, M.; Clarke, D. B. (1999): Hearing places, making spaces: sonorous geographies, ephemeral rhythms, and the Blackburn warehouse parties. In: Environment and Planning D 17 (3), 283-305.

Ingold, T.; Hallam, E. (2007): Creativity and cultural improvisation: an introduction. In: Hallam, E.; Ingold, T. (Hrsg.): Creativity and cultural improvisation. Oxford, 1-24.

Jackson, P. (2000): Rematerializing social and cultural geography. In: Social \& Cultural Geography 1 (1), 9-14.

Jacobs, J. (1961): The death and life of great American cities. New York.

Jayne, M.; Gibson, C.; Waitt, G.; Bell, D. (2010): The cultural economy of small cities. In: Geography Compass 4 (9), 1408-1417.

Kirchberg, V. (2010): Kreativität und Stadtkultur: stadtsoziologische Deutungen. In: Hannemann, C.; Glasauer, H.; Pohlan, J.; Pott,
A.; Kirchberg, V. (Hrsg.): Jahrbuch StadtRegion 2009/2010: Stadtkultur und Kreativität. Opladen, 19-44.

Krätke, S. (2011): The creative capital of cities: interactive knowledge creation and the urbanization economies of innovation. Chichester.

Kunzmann, K. R. (1992): Kultur, Wirtschaft und Raumentwicklung. In: Informationen zur Raumentwicklung 4/5, 185-197.

Landry, C. (2000): The creative city: a toolkit for urban innovators. London.

Lange, B.; Bürkner, H.-J. (2010): Wertschöpfung in der Kreativwirtschaft: der Fall der elektronischen Klubmusik. In: Zeitschrift für Wirtschaftsgeographie 54 (1), 46-68.

Mager, C. (2000): Kommunale Kulturpolitik und lokale Kulturarbeit: Dynamik und Entwicklungen des soziokulturellen Zentrums ,Karlstorbahnhof ${ }^{*}$ in Heidelberg. In: Journal der Heidelberger Geographischen Gesellschaft 15, 164-183.

Marcuse, H. (1965): Kultur und Gesellschaft. 2 Bände. Frankfurt am Main.

Markusen, A. (2006): Urban development and the politics of a creative class: evidence from a study of artists. In: Environment and Planning A 38 (10), 1921-1940.

Mitscherlich, A. (1965): Die Unwirtlichkeit unserer Städte. Frankfurt am Main.

Molotch, H. (2002): Place in product. In: International Journal of Urban and Regional Research 26 (4), 665-688.

Mossig, I. (2009): Zentralisierungstendenzen in der Medienwirtschaft: zur Bedeutung des räumlichen Kontextes für die Erzeugung und kommerzielle Verwertung von Medieninhalten. In: Geographische Zeitschrift 97 (2/3), 95-112.

MWMTV - Ministerium für Wirtschaft und Mittelstand, Technologie und Verkehr des Landes Nordrhein-Westfalen (Hrsg.) (1998): 3. Kulturwirtschaftsbericht. Kulturwirtschaft in Nordrhein-Westfalen: Kultureller Arbeitsmarkt und Verflechtungen. Düsseldorf.

Olma, S. (2009): Kritik der Kreativindustrien. In: Lange, B.; Kalandides, A.; Stöber, B.; Wellmann, I. (Hrsg.): Governance der Kreativwirtschaft: Diagnosen und Handlungsoptionen. Bielefeld, $103-120$.

Peck, J. (2005): Struggling with the creative class. In: International Journal of Urban and Regional Research 29 (4), 740-770.

Power, D.; Hallencreutz, D. (2002): Profiting from creativity? The music industry in Stockholm, Sweden and Kingston, Jamaica. In: Environment and Planning A 34 (10), 1833-1854.

Pratt, A. C. (2008): Creative cities: the cultural industries and the creative class. In: Geografiska Annaler, Series B: Human Geography 90 (2), 107-117.

Pratt, A. C. (2011): The cultural contradictions of the creative city. In: City, Culture and Society 2 (3), 123-130.

Rammstedt, O.; Wagner, G. (1998): Neue soziale Bewegungen. In: Schäfers, B.; Zapf, W. (Hrsg.): Handwörterbuch zur Gesellschaft Deutschlands. Bonn, 483-486.

Rantisi, N. M.; Leslie, D. (2010): Materiality and creative production: the case of the Mile End neighborhood in Montréal. In: Environment and Planning A 42 (12), 2824-2841.

Reckwitz, A. (2013): Die Erfindung der Kreativität. In: Kulturpolitische Mitteilungen, 141 (2), 23-34.

Scott, A. J. (2000): The cultural economy of cities: essays on the geography of image-producing industries. London.

Scott, A. J. (2010): Cultural economy and the creative field of the city. In: Geografiska Annaler, Series B: Human Geography 92 (2), $115-130$.

Shaw, K. (2013): Independent creative subcultures and why they matter. In: International Journal of Cultural Policy 19 (3), 333-352.

Sievers, N.; Wagner, B. (Hrsg.) (1992): Bestandsaufnahme Soziokultur: Beiträge, Analysen, Konzepte. Stuttgart.

Strittmatter, T. (1992): Tendenzen und Probleme der Umgestaltung der kulturellen Infrastruktur in den neuen Ländern. In: Informationen zur Raumentwicklung 1, 11-18. 
Taubmann, W. (1987): Städtische Kulturangebote als raumwirksamer Wirtschaftsfaktor: das Beispiel Bremen. In: Berichte zur deutschen Landeskunde 61 (1), 147-172.

von Beyme, K. (2012): Kulturpolitik in Deutschland: von der Staatsförderung zur Kreativwirtschaft. Wiesbaden.

Waitt, G.; Gibson, C. (2009): Creative small cities: rethinking the creative economy in place. In: Urban Studies 45 (5-6), 1223-1246.
Watson, A.; Hoyler, M.; Mager, C. (2009): Spaces and networks of musical creativity in the city. In: Geography Compass 3 (2), 856-878.

Weckerle, C.; Söndermann, M.; HfGK - Hochschule für Gestaltung und Kunst Zürich (2003): Erster Kulturwirtschaftsbericht Schweiz: Das Umsatz- und Beschäftigungspotential des kulturellen Sektors. Zürich. 